\title{
Dissolved organic matter percolated from periphyton in the Everglades: Characterization and interaction with mercury \\ Afia Anjuman ${ }^{1}$, Yong Cai ${ }^{*}$
}

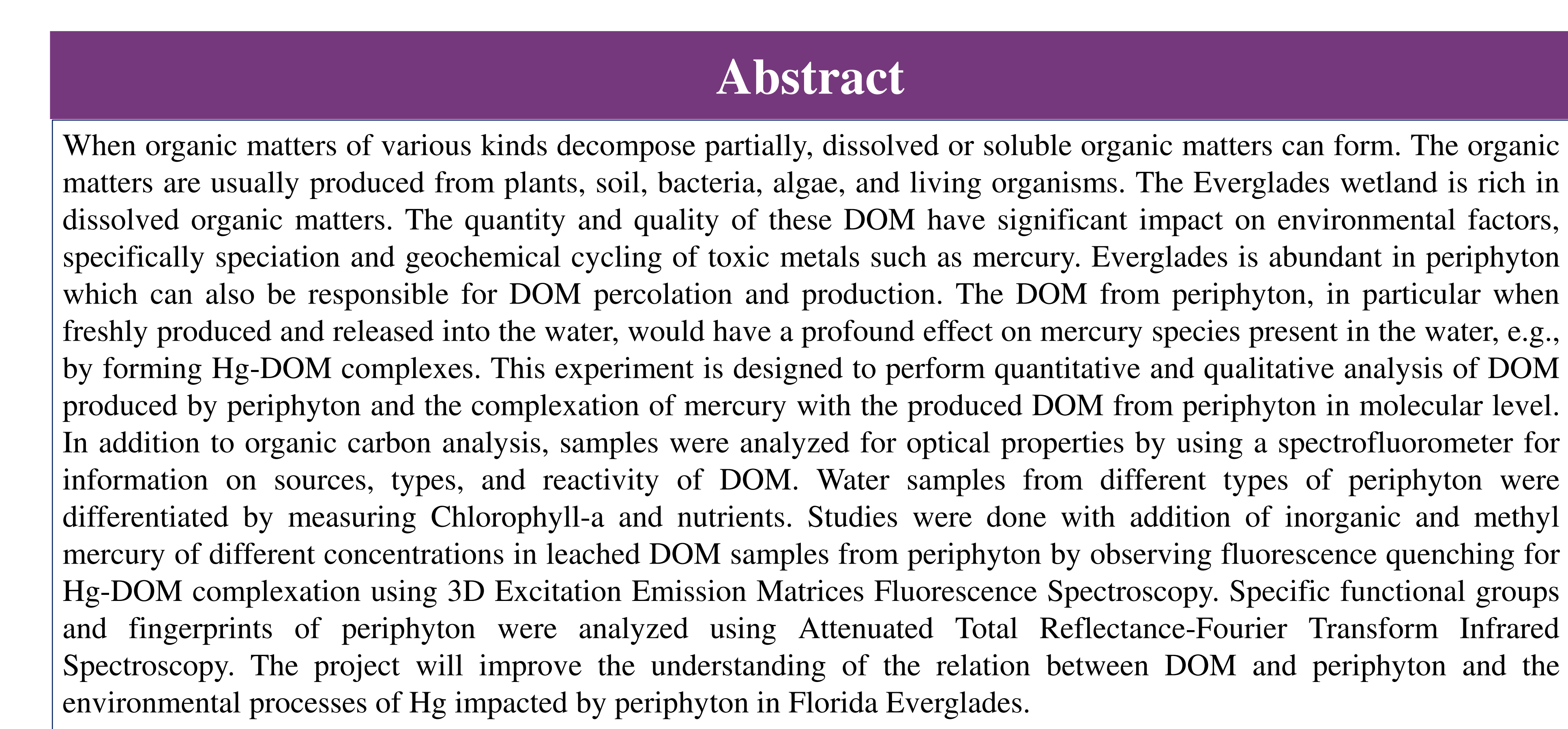

Research aims

$>$ Quantitative and qualitative characterization of DOM from different types of periphyton and in Everglades surface water

$>$ Analysis of periphyton DOM-mercury interaction through addition of different concentrations of mercury in freshly leached DOM samples from periphyton
$>$ Characterization of specific functional groups and fingerprints of periphyton organic matter which interact with mercury

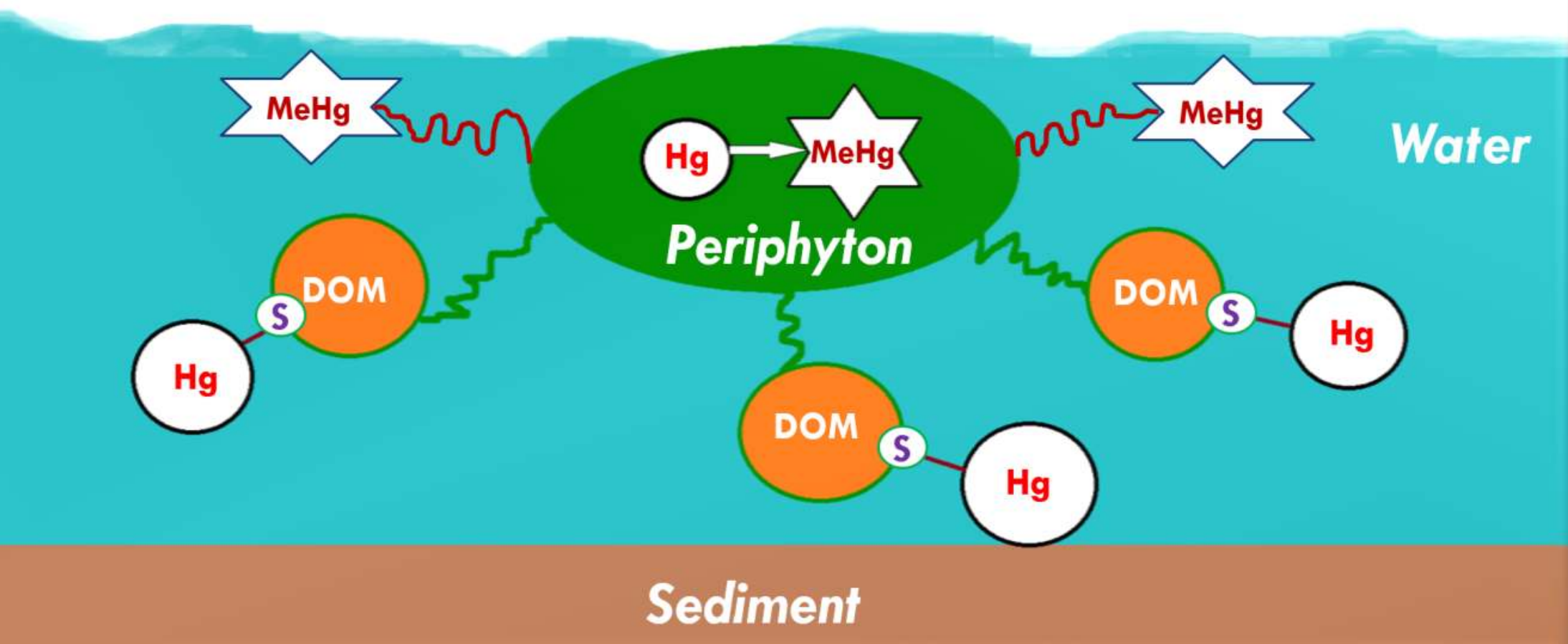

Figure 1: Periphyton dissolved organic matter impacting mercury speciation in Florida Everglades Research Methodology

> Sample collection of surface water and different types of periphyton from Everglades
Incubation of periphyton and organic matter leaching experiment
$>$ Elemental analysis by measuring total and dissolved C, N, P contents

$>$ Optical properties measurement without and with mercury of different concentrations using 3-D

EEMs Fluorescence Spectroscopy

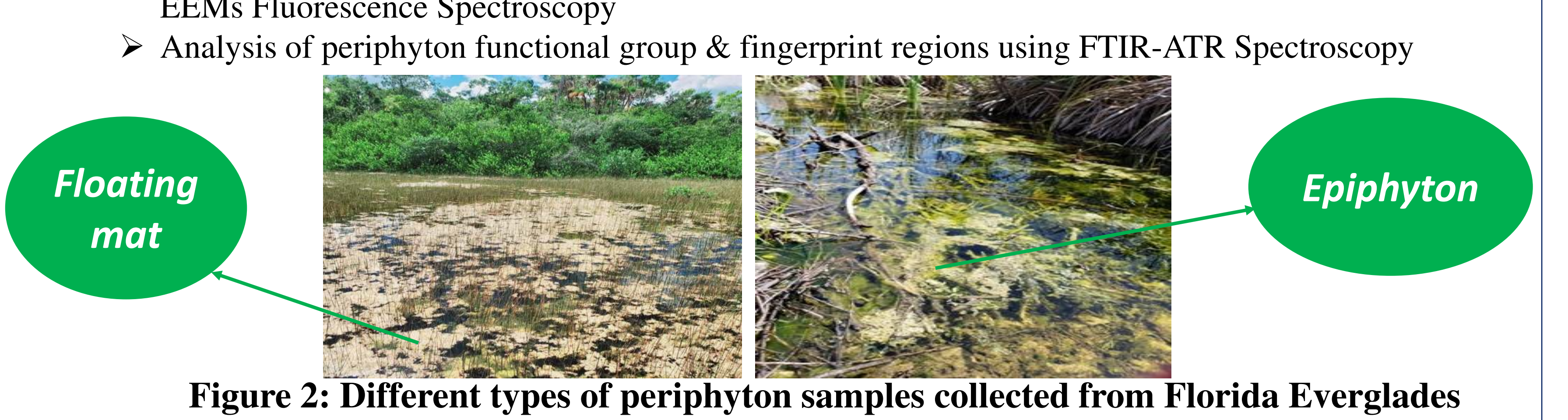

Figure 2: Different types of periphyton samples collected from Florida Everglades

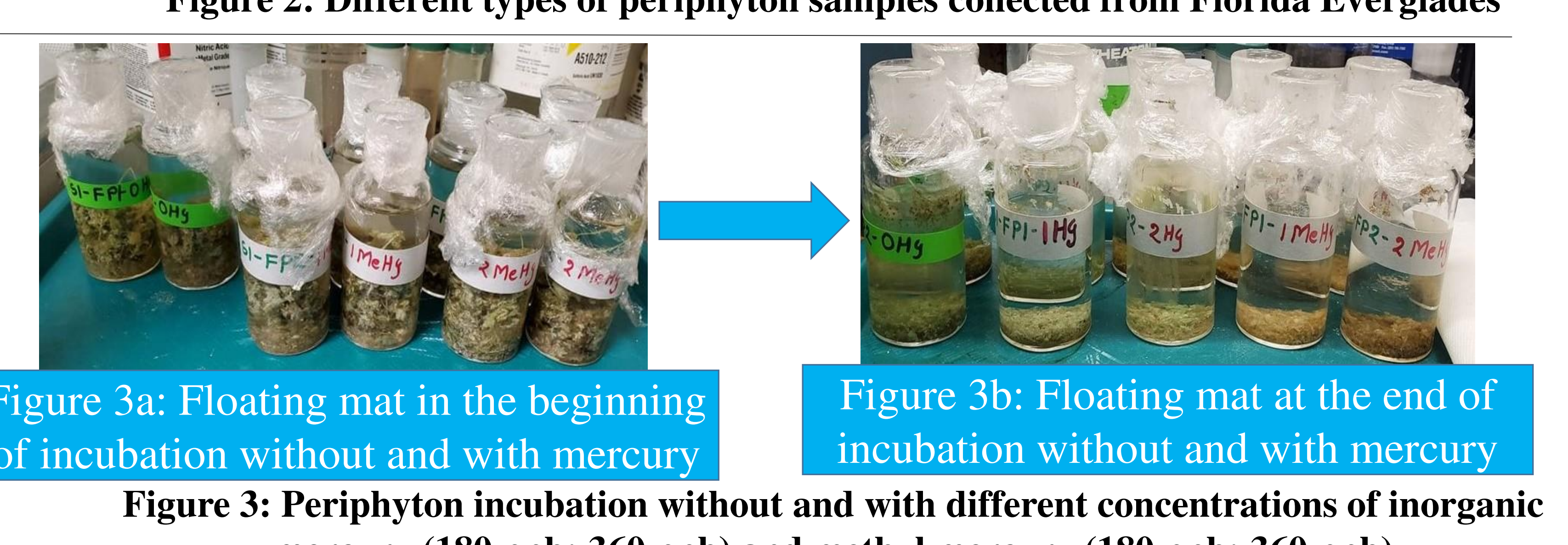

Figure 3: Periphyton incubation without and with different concentrations of inorganic mercury (180 ppb; 360 ppb) and methyl mercury (180 ppb; 360 ppb)

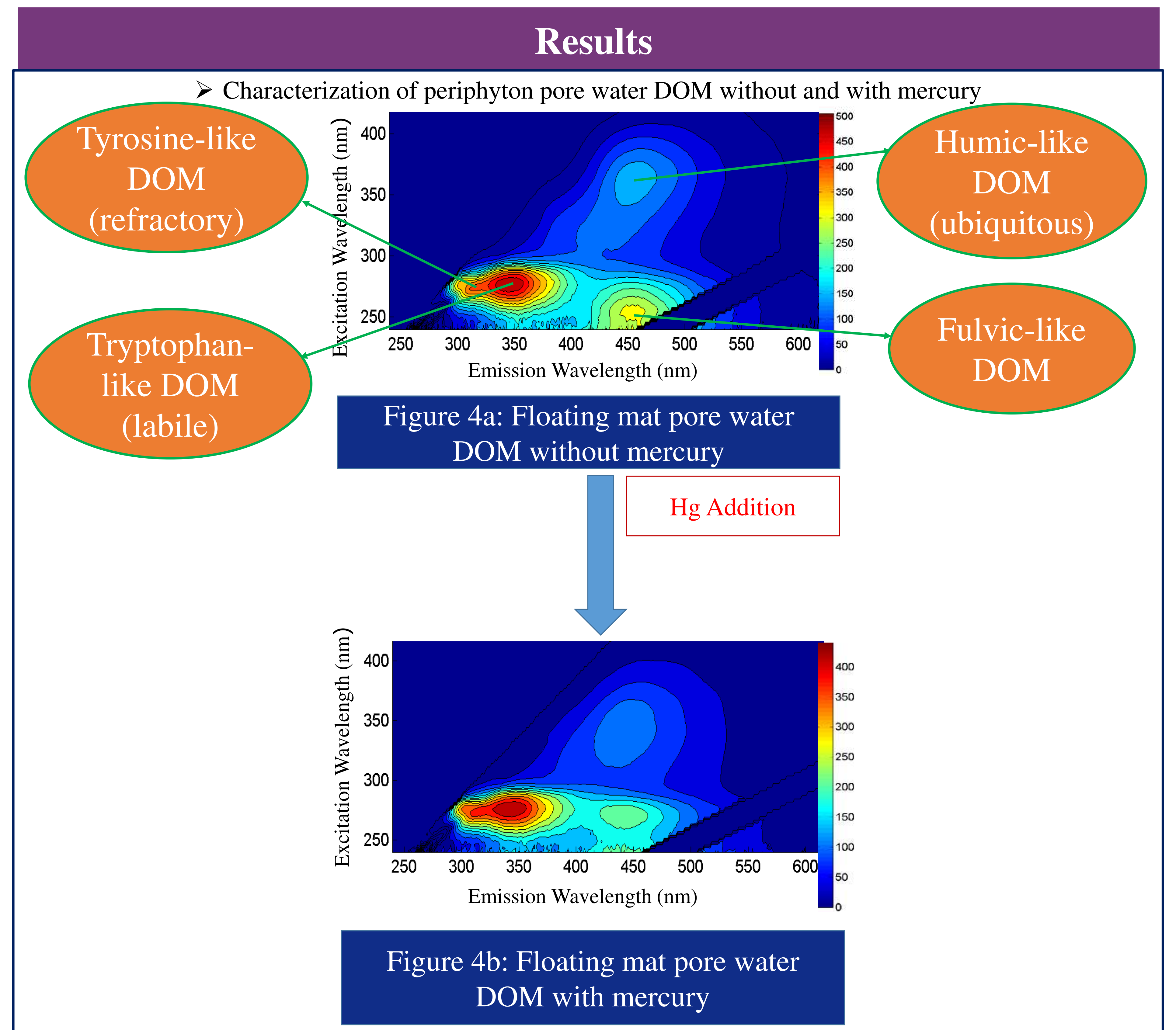

Figure 4: Periphyton DOM optical properties analysis without and with inorganic mercury (360 ppb) using 3D EEMs Fluorescence Spectroscopy

$>$ Characterization of periphyton organic matter functional group \& fingerprint regions

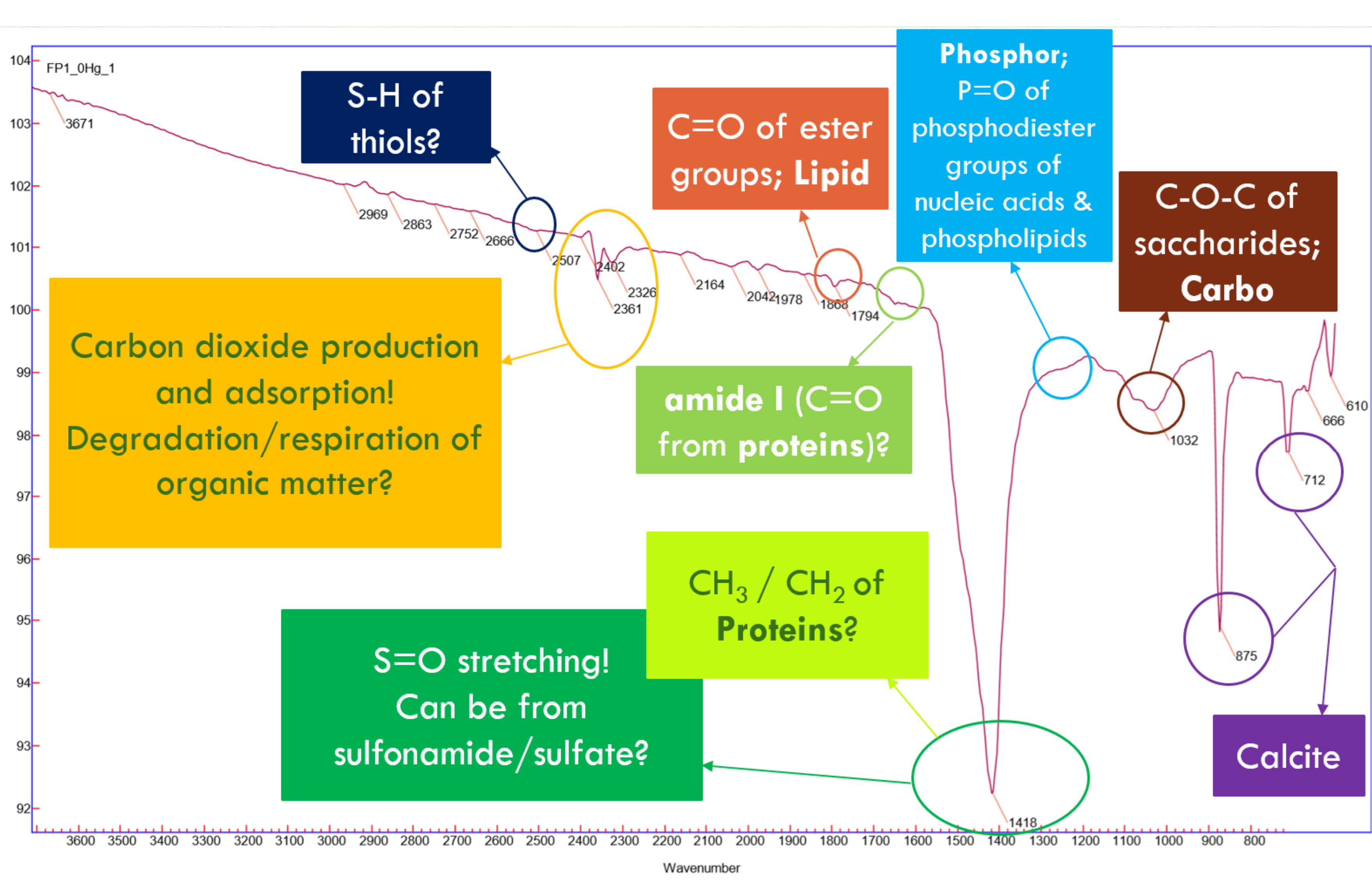

Figure 5: Periphyton functional group \& fingerprint regions analysis using FTIR-ATR Spectroscopy

\section{Findings and Observations}

DOM types from floating mat pore water were mostly tryptophan-like (labile), followed by tyrosine-like (refractory), then fulvic-like, and lastly humic-like (ubiquitous origin)

Addition of inorganic mercury of $360 \mathrm{ppb}$ had
indicating periphyton DOM-Hg complexation

Floating mat fingerprint region exhibited the stron

Floating mat fingerprint region exhibited the strong
of $\mathrm{COO}^{-}$groups and sulfate or, sulfonamide group

C-O-C of saccharides, calcite, amide I ( $\mathrm{C}=\mathrm{O}$ from proteins), lipid ( $\mathrm{C}=\mathrm{O}$ of ester), phosphor ( $\mathrm{P}=\mathrm{O}$ of phosphodiester) and low absorbance of thiol (S-H) functional group were observed as well

\section{Discussion and Conclusion}

In this project, quantitative and qualitative analysis of dissolved organic matters were performed for Everglades surface water, periphyton overlying water, and periphyton pore water from two different types

of periphyton.
The quantitative analysis yielded information on their dissolved organic carbon, total dissolved nitrogen unique environmental conditions, light, oxygen availability, nutrient uptake and photosynthetic abilities: while the qualitative analysis vielded information on their unique DOM types, sources, and reactivity. In order to analyze the influence of mercury in periphyton DOM, the most abundant and common floating mats were utilized by adding different concentrations of inorganic and methyl mercury in their pore water which provided information on mercury's unique ability to complex to different periphyton DOM types. Floating mats were also utilized to obtain information on periphyton functional group and fingerprint regions without and with incubation with mercury. Although, there were no significant differences obtain among the absorbce qualitative information regarding their fingerprint regions were made.

mats by incubating them without and with different concentrations of inas also analyzed by using floating 's ability to inhibit photosynthetic ability of periphyton. Acknowledgements

This material is based upon work supported by the National Science Foundation under Grant No. HRD1547798. This NSF Grant was awarded to Horida International University as part of the Centers of Research Excellence in Science and Technology (CREST) Program. Any opinions, findings, and necessarily reflect the views of the National Science Foundation

My advisor and committee members: Dr. Yong Cai, Dr. Kevin O'Shea, Dr. Bruce McCord, Dr. Piero Gardinali, and Dr. Evelyn Gaiser

Dr. Rudolf Jaffe for DOM and Fluorescence Workshop 2018

5. Dr. Guangliang Liu and lab mates in Dr. Yong Cai's research lab
5. Dithe

\section{References
R. Dr. Guangliang Liu and lab mates in Dr. Yong Ca's research}

Hagerthey, S. E;; Bellinger, B. J.; Wheeler, K.; Gantar, M.; Gaiser, E., Everglades periphyton: a biogeochemical

perspective. Critical Reviews in Environmental Science and Technology 2011, 41 (S1), 309-343.
Jiang, P.; Liu, G.; Cui, W.; Cai, Y., Geochemical modeling of mercury speciation in surface water and implication
on mercury cycling in the everglades wetland. Science of The Total Environment 2018 , 640, 454-465.

3. Maie, N.; Jaffe, R.; Miyoshi, T.; Childers, D. L., Quantitative and qualitative aspects of dissolved organic carbon

leached from senescent plants in an oligotrophic wetland. Biogeochemistry 2006, 78 (3), 285-314.
Liu, G.: Cai, Y.: Philippi, T.; Kalla, P.; Scheidt, D.; Richards, J.; Scinto, L.: Appleby, C., Distribution of total and Liu, G.; Cai, Y.; Philippi, T.; Kalla, P.; Scheidt, D.; Richards, J.; Scinto, L.; Appleby, C., Distribution of total and
methylmercury in different ecosystem compartments in the Everglades: Implications for mercury bioaccumulation

Environmental Pollution 2008, 153 (2), 257-265.

Hansen, A. M.; Kraus, T. E.; Pellerin, B. A.; Fleck, J. A.; Downing, B. D.; Bergamaschi, B. A., Optical properties dissolved organic matter (D.

Lu, X.; Jaffe, R., Interaction between $\mathrm{Hg}$ (II) and natural dissolved organic matter: a fluorescence spectroscopy

based study. Water Research 2001, 35 (7), 1793-1803.

Stehfest, K.; Toepel, J.; Wilhelm, C., The application of micro-FTIR spectroscopy to analyze nutrient stress-related

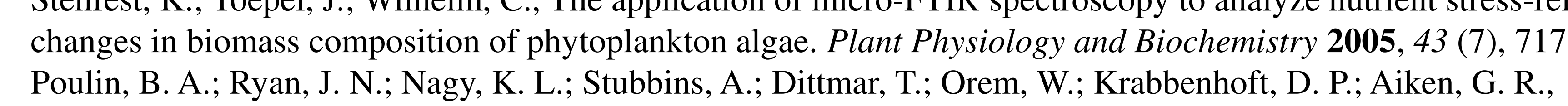

Spatial dependence of reduced sulfur in Evergladedes dissolved organic matter controlled by sulfate enrichment.

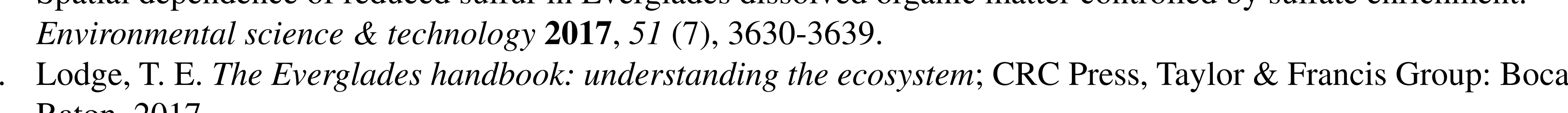

